\title{
Técnicas de análisis de posicionamiento
}

Por Luis Cueto Álvarez de Sotomayor, Chimo Soler Herreros, Raquel Entremonzaga Ayuso, Eva Martínez, Antonio Carrasco, Isidro Aguillo, Elena FernánArtículo dez, Miguel Rodríguez Artacho, Dionisio Barrantes, Javier Espadas Bardón, Rufino Ferreras Marcos, Jaime Albert, Jesús Sanz de las Heras

Luis Cueto Álvarez de Sotomayor, licenciado en derecho, pertenece desde el año 1987 al Cuerpo Superior de Administradores Civiles del Estado. Ha desempeñado diversos puestos en los departamentos de Trabajo, Justicia o Exteriores (Instituto Cervantes). En la actualidad ocupa el puesto de jefe del Gabinete Técnico de la Subsecretaría del Ministerio de Industria, Turismo y Comercio.

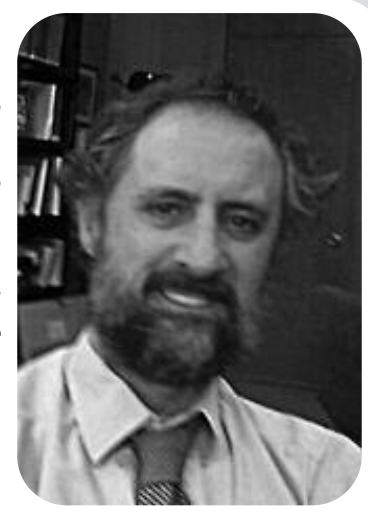

Eva Martínez, diplomada en informática por la Universidad Pontificia de Salamanca (1990). Es jefa de informática y responsable de nuevas tecnologías de la Casa de América, funciones que desempeña desde 1992. Ha planificado y desarrollado varios proyectos en dicha institución, a destacar la digitalización audiovisual y organización informática de todos los actos que se celebran en la Casa de América. Actualmente continúa presentando nuevos proyectos y trabajando junto con el equipo de posicionamiento en la elaboración de estrategias para internet.

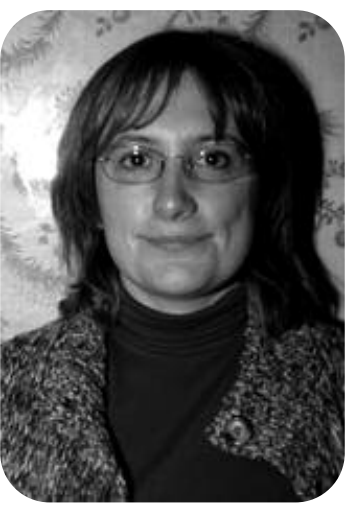

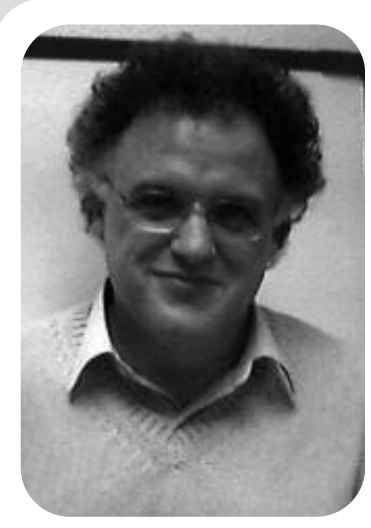

Chimo Soler Herreros, licenciado en historia contemporánea por la Universitat de València. Fue coordinador técnico en la sede valenciana de la Universidad Internacional Menéndez Pelayo y desde 1991 es jefe de Sistemas de Información del Instituto Cervantes. Ha publicado varios artículos y comunicaciones en congresos sobre historia, lenguas y culturas en internet. Colabora habitualmente con varias universidades, especialmente con las universidades de Valencia y Alicante en proyectos en internet y coordina desde el año 2002 un proyecto de investigación sobre el posicionamiento en internet de instituciones culturales, científicas y educativas.
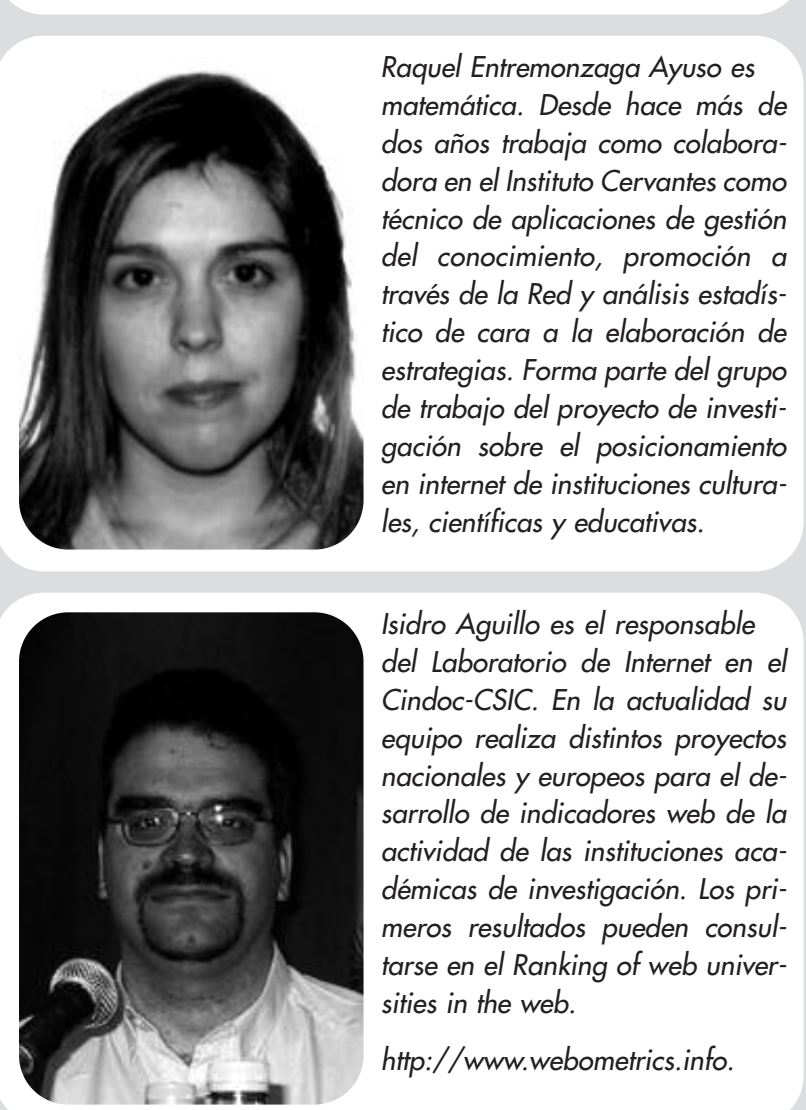

Isidro Aguillo es el responsable del Laboratorio de Internet en el Cindoc-CSIC. En la actualidad su equipo realiza distintos proyectos nacionales y europeos para el desarrollo de indicadores web de la actividad de las instituciones académicas de investigación. Los primeros resultados pueden consultarse en el Ranking of web universities in the web.

http://www.webometrics.info.

Resumen: El presente artículo propone una serie de técnicas orientadas al estudio del posicionamiento en internet de una sede web. Dichas técnicas deben proporcionar al analista información suficiente para poder realizar interpretaciones conducentes a la adecuada elección de estrategias eficaces y rentables de mejora.

Palabras clave: Posicionamiento, Marketing, Buscadores, Cultural, Contenidos, Servicios, Enlaces, Visibilidad, Sociabilidad. 
Miguel Rodríguez Artacho, ingeniero en informática por la UPM (1994) y doctor ingeniero industrial por la UNED (2000) es director de planificación de sistemas informáticos y profesor titular de la Universidad $\mathrm{Na}$ cional de Educación a Distancia. Ha trabajado desde 1999 como consultor externo para el comité de estándares CEN/ISSS LTWS de tecnologías educativas y para los proyectos IST Celebrate y Digicult del 50 PM de la UE. Desde 2003 trabaja también en el desarrollo de estrategias de posicionamiento de contenidos educativos en internet en la UNED.

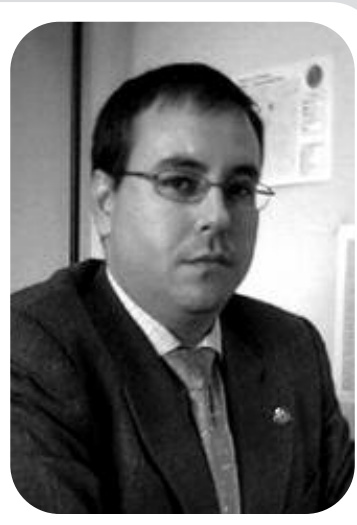

Elena Fernández, trabaja en el Centro de Información y Documentación Científica (CindocCSIC). Es coordinadora de la sección de portales científicos (Tecnociencia, Hispagua, Salut). Actualmente es responsable de varios proyectos de investigación del VI Programa Marco y de la Comu nidad Madrid.

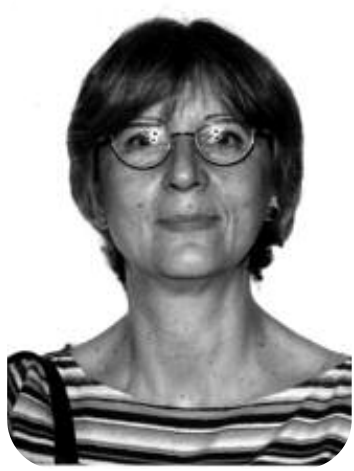

Title: Analytic techniques for positioning

Abstract: The present article proposes a series of techniques oriented to the study of positioning a headquarters web in the internet. These techniques must provide sufficient information to allow the analyst to make judgements conducive to the appropriate selection of effective and productive strategies for improvement.

Keywords: Positioning, Marketing, Seekers, Cultural, Contents, Services, Links, Visibility, Sociableness

Cueto Álvarez de Sotomayor, Luis; Soler Herreros, Chimo; Entremonzaga Ayuso, Raquel; Martínez, Eva; Carrasco, Antonio; Aguillo, Isidro; Fernández, Elena; Rodríguez Artacho, Miguel; Barrantes, Dionisio; Espadas Bardón, Javier; Ferreras Marcos, $R$ ufino; Albert, Jaime; Sanz de las Heras, Jesús. «Técnicas de análisis de posicionamiento». En: El profesional de la información, 2004, enero-febrero, v. 14, n. 1, pp. 14-20.

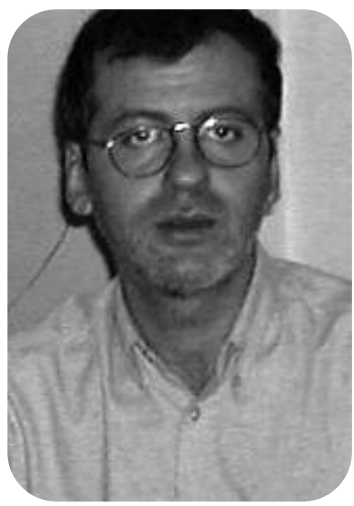

Rufino Ferreras Marcos, responsable del Departamento de Desarrollo Edu cativo del Museo ThyssenBornemisza, coordinador de la web EducaThyssen, ha participado en la producción de varios cd-rom y portales sobre educación artística y es autor de varios libros y artículos sobre educación y tecnologías de la información.

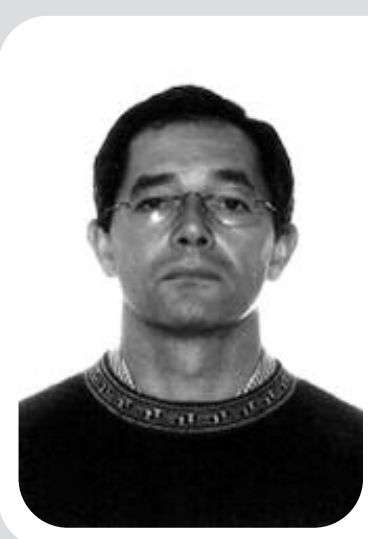

Dionisio Barrantes, jefe de servicio de desarrollos informáticos, coordinador del equipo encargado del mantenimiento y desarrollo del catálogo automatizado Ariadna, del mantenimiento de sitio web de la Biblioteca Nacional y del desarrollo de nuevos servicios web que faciliten la difusión de los fondos custodiados por la biblioteca.

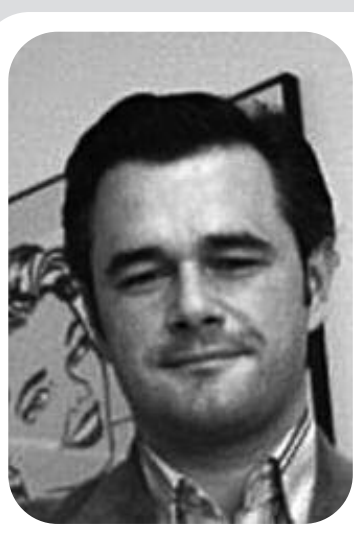

Javier Espadas Bardón, MBA Instituto de Empresa. Jefe del Departamento de Informática de la Fundación Colección ThyssenBornemisza. Viene desarrollando desde el año 2002 un proyecto de investigación sobre el posicionamiento en internet de instituciones culturales, científicas y educativas.

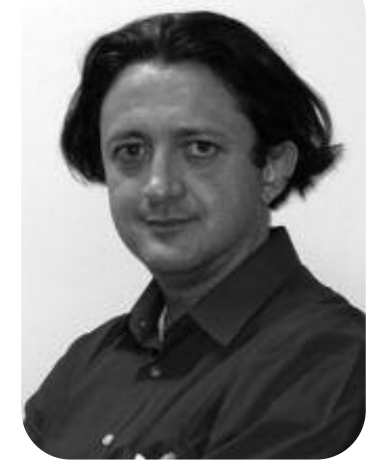

Jaime Albert, licenciado en filosofía y letras y MBA. Tras su paso por Antena 3 Televisión, Antena 3 Interactiva y AOL Time Warner, en la actualidad es responsable de comunicación de la Entidad Pública Empresarial Red.es.

Jesús Sanz de las Heras es coordinador del servicio de correo electrónico de Redlris y coordinador del Servicio de Redlris de Redes Temáticas.

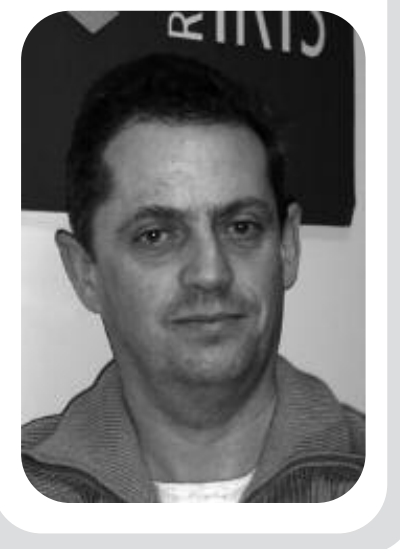

\section{Introducción}

Debido al exponencial crecimiento de internet, que cuenta actualmente con varios miles de millones de páginas, es necesario a la hora de publicar, mantener y desarrollar un sitio, prestar atención a su posicionamiento. Este concepto, nacido en el mundo del marketing convencional, se viene aplicando cada vez con mayor frecuencia al digital, si bien con algunas diferencias y matizaciones. 
Por ejemplo, el posicionamiento de los iconos culturales en internet no siempre es un reflejo de la realidad de un sitio. Si buscamos «Velázquez Prado Museum» (figura 1) o «Velázquez Louvre» (figura 2) en Google, de entre unos 7.000 y 8.000 resultados que aparecen, respectivamente, no se encuentran enlaces directos a los portales institucionales de dichos museos entre las 10 primeras posiciones, como sería lo natural. En general, los contenidos, audiencias y resultados de un portal cultural no se corresponden con los contenidos y públicos de su actividad presencial cotidiana.

El posicionamiento en Internet ha sido definido como la posición que ocupa un portal, desde el punto de vista de los usuarios, en relación con su competencia No debe reducirse únicamente al campo de acción de los buscadores (como es costumbre), sino que guarda relación también con otros cauces de la navegación por la Red (como el correo electrónico o los enlaces establecidos entre sitios) que, si bien tienen generalmente un menor peso cuantitativo, pueden llegar a adquirir un valor cualitativo considerablemente mayor.

Esta necesidad ha movido a diferentes investigadores y especialistas a unir sus conocimientos y experiencias para tratar de consensuar un vocabulario técnico de unidades e indicadores, un método global de análisis y una serie de criterios de interpretación.

El presente artículo muestra uno de esos intentos de definición de unas técnicas globales para el análisis del posicionamiento de un sitio en internet.

\section{Objetivos}

En junio de 2003, tras una primera puesta en común de los puntos de vista de diferentes entidades, se formó un grupo de trabajo coordinado por el Instituto Cervantes y formado por instituciones con presencia en la Red, como la UNED, el Cindoc-CSIC, Red.es, el Museo Thyssen, la Casa de América, la Biblioteca Nacional de España o la Biblioteca Virtual Miguel de

Eduard Maner

\section{Figura 1}

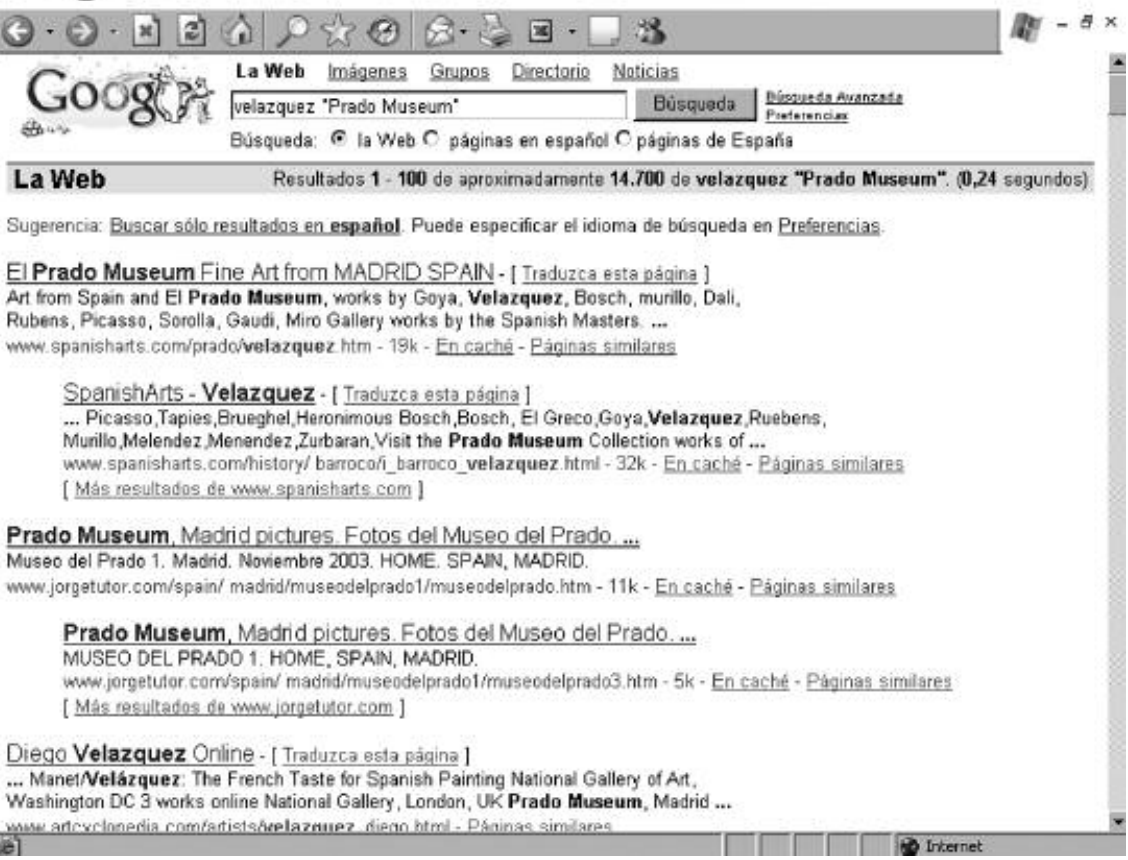

\section{Figura 2}

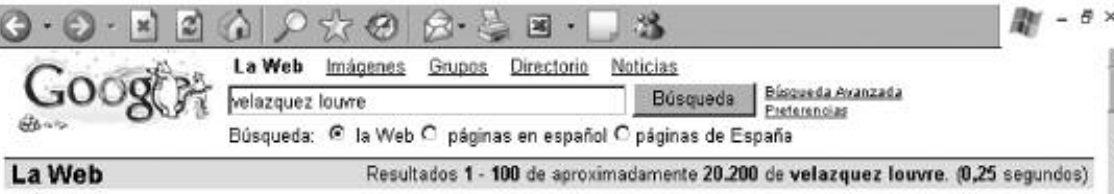

Sugerencia. Euscar sỏla resultados an español Puede especificar el idioma de búsqueda en Preferencias.

Diego de Silva y Velazquez

(a)

Museo del Prado), y la jonen Infarna Maria Teresa (Museo del Louvre y Museo ...

whw iespana esflegislaciones/Velazquez. $\mathrm{hm}$ - $14 \mathrm{k}$ - En cachí - Paginas similanes

... Los museos fueron su verdadera escuela: en el Louvre copio los "Pequeños caballeros".

mostrando un gran interes por la pintura espaniola y...

[Más resultados de waweriaspana es]

Galeria de arte del Ergonine

... Museum, El aguador de Serilla Diego Rodtiguez de Siva Velázquez Palazzo Pitti ... The

Tooih Puller Gerrit van Horithorst (1628) Museo del Lourre, The Quackdocter ...

作

Diego Velázquez. Diego Rodriguez de Silva y Velázquez. El aguador de Serilla. Lienzo. ...

Como he supuesto en mi libro Velázquez en Sevilla (1974, pág ...

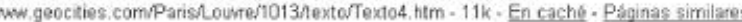

SMP. Diego velazquez

que lo pintase en italia además de ciertas influencias, más o menos dudosas, del

Hermafrodita Borghese (hoy en el Louvre) del que mandó Velázquez hacer un..
Cervantes. Dicho equipo de trabajo contó, asimismo, desde un principio con el apoyo de RedIris, que creó una lista de distribución denominada Posito para el intercambio de información relacionada con la temática analizada.

Con el trabajo de dicho grupo y de todos los eventos organizados en torno a la voluntad del desarrollo del estudio del posicionamiento en internet, así como la creciente necesidad de realizar encuentros institucionales orientados al intercambio de información y experiencias junto con la puesta en común de métodos y estrategias, se desarrolla una metodología común pa- 
ra la consecución del objetivo, donde se recoge la investigación realizada por los firmantes del presente artículo y se sistematizan los estudios de casos presentados en Alicante, en las jornadas Posicionamiento.es, analizando los siguientes puntos:

- Los objetivos estratégicos de cada sitio, áreas de actividad, socios principales, público objetivo y su agenda de actividades.

- Los contenidos del sitio, realizando un estudio de sus materiales, de los servicios e informaciones que son publicados en el mismo: volumen ofrecido, estructura, funcionalidades, glosario de términos más usados, prestaciones multilingües y enlaces recomendados.

-El uso del sitio a través de los datos internos y de las encuestas externas para conocer su utilidad, los sitios que lo recomiendan, los usuarios que lo visitan, la duración y la procedencia de las visitas y su efecto sobre la actividad presencial de cada sitio.

- Su popularidad, su ubicación temática en la Red y la integración en redes de usuarios, los sitios que remiten y recomiendan el portal, la difusión del mismo por distintos medios y su efecto sobre el incremento de las visitas, la visibilidad en los buscadores y su eficacia.

- Análisis de otros portales dentro del ámbito temático del sitio que se encuentren mejor posicionados en la Red.

-Estudio de la eficiencia y de la rentabilidad mediante la comparación de los objetivos estratégicos de cada sitio con los resultados obtenidos tras la elaboración de las estrategias descritas.

\section{Análisis de los objetivos estratégicos}

Se definen los objetivos estratégicos del sitio para conseguir que tengan su reflejo en internet. Para ello,

\section{Figura 3}

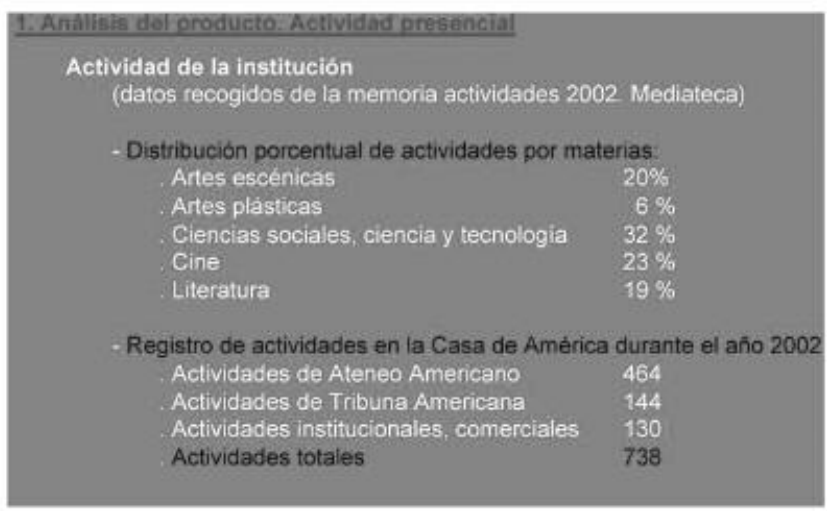

se analizan el ámbito temático (descriptores por campos de actividad), los socios o colaboradores, los términos o palabras clave que definen las actividades o servicios del sitio y se esteblece el público objetivo. Un ejemplo puede verse en la figura 3 la Casa de América (para más información remitimos a la correspondiente ponencia del curso sobre posicionamiento durante las jornadas de Estrategias de posicionamiento en internet, que tuvo lugar en el mes de noviembre de 2003 en la Casa de América).

\section{Análisis de los contenidos del sitio}

- Se realiza un estudio de los materiales, de los servicios e informaciones que son publicados: volumen de los contenidos, estructura, funcionalidades, glosario de términos más usados, prestaciones multilingües y enlaces recomendados.

\section{«El posicionamiento en internet ha sido definido como la posi- ción que ocupa un sitio, desde el punto de vista de los usua- rios, en relación con su compe- tencia»}

- Se mide su calidad, estudiando si cumple los estándares relacionados con la accesibilidad y la usabilidad del mismo. A través de un mantenimiento periódico de las páginas se analizan los posibles enlaces rotos, que son aquellos que por algún motivo no son alcanzables a través de la web y que conviene corregir.

- Se mide el volumen de los contenidos de las páginas, así como los servicios que ofrece el sitio, verificándose que el formato de las mismas sea el adecuado para su indexación en los distintos buscadores.

- Se mide la variedad de los idiomas utilizados y los servicios personalizados.

Todo esto se realiza mediante el uso de herramientas de rastreo de portales que proporcionan los indicadores básicos de un sitio: número, tamaño, tipo de sus páginas, mapa web, palabras más frecuentes de las páginas visibles y profundidad de la estructura.

Se recomienda realizar un análisis de las páginas más visitadas, estudiando aquellas con más y menos accesos de usuarios y analizando la visibilidad de los contenidos del sitio.

\section{Análisis del uso del sitio}

-A partir de los ficheros .log, se analizan las tendencias de los usuarios, es decir, el volumen de las páginas más visitadas, el número de visitas, su duración y procedencia, al igual que su efecto sobre la actividad presencial de cada sitio.

El profesional de la información, v. 14, n. 1, enero-febrero 2005 


\section{Figura 4}

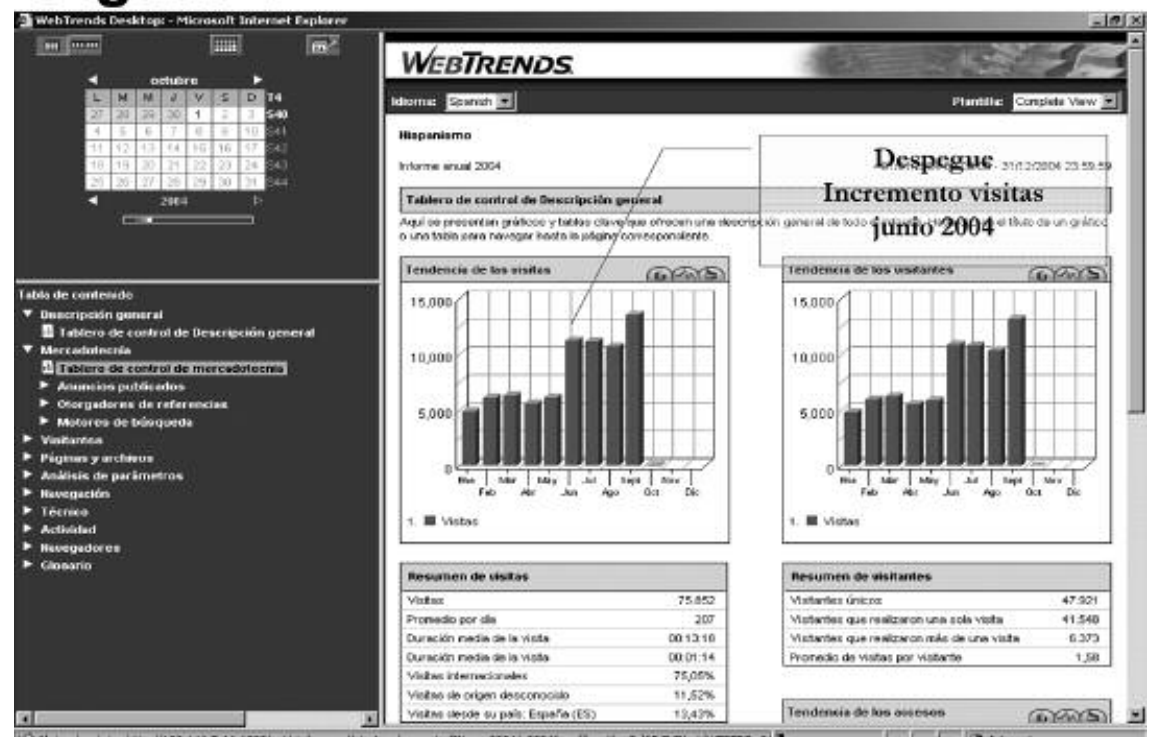

\section{Figura 5}

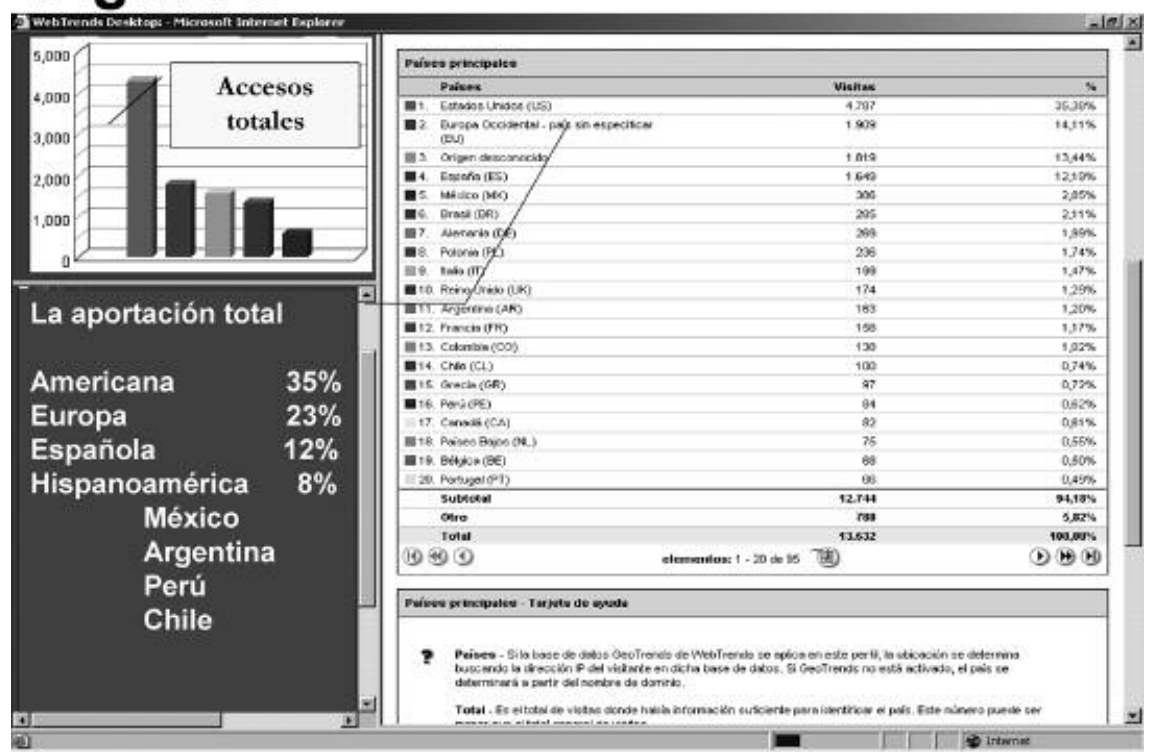

\section{Figura 6}

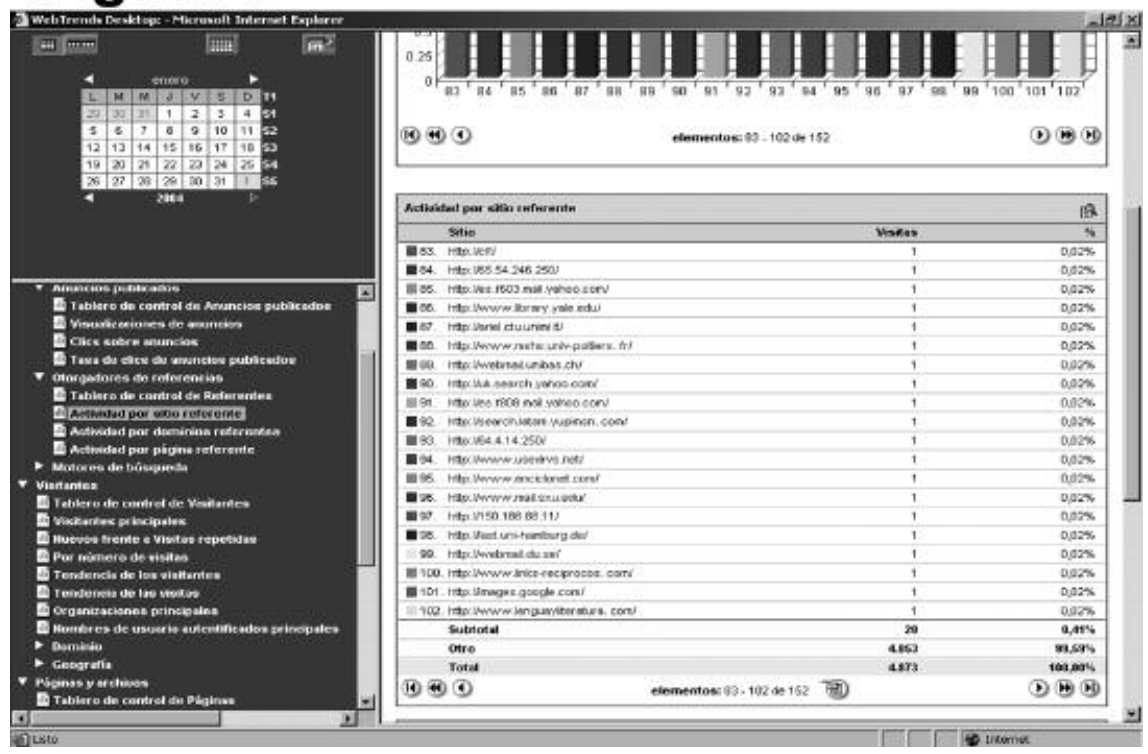

- Se compara el volumen (número de visitas), la calidad (páginas vistas por un usuario) y su tiempo de permanencia en el sitio (figura 4).

- Se analizan las páginas más vistas y demandadas por el mayor número de usuarios.

- Se estudia la procedencia de las visitas, los países de origen, teniendo en cuenta que la mayoría de herramientas de análisis, usan el dominio de primer nivel de procedencia en lugar de la dirección IP y asignan arbitrariamente a EUA los usuarios de los dominios .com. (figura 5).

- Se analizan los motivos y las vías de acceso al sitio: desde buscadores, correo-e, enlaces desde otros sitios, accesos directos, etc., así como las costumbres de navegación (figura 6).

Para todo esto se recomienda hacer uso de un programa de análisis de tráfico web y de una encuesta así como conocer las limitaciones de los analizadores y los márgenes de error de las encuestas a la hora de realizar interpretaciones y valoraciones.

\section{Análisis de la popularidad del sitio}

Se trata de analizar sus factores de éxito: servicios de contenidos, visibilidad y posicionamiento en los buscadores, vecindario temático, sociabilidad, integración en redes y difusión en los medios de comunicación. También conviene realizar un análisis de otros sitios con objetivos o públicos similares o que alojen contenidos relacionados para detectar ideas innovadoras y que sirva de guía para una mejora del posicionamiento. Esto permitirá establecer alianzas estratégicas entre ellos.

Se estudia la procedencia de los referidores, el ranking de páginas de Google (pagerank), el número de enlaces externos e internos y la 
popularidad (posición en el ranking).

La página Alexa del grupo Amazon, nos devuelve un ranking de sedes a nivel mundial; es decir, dispone de un censo superior a los diez millones de páginas y en función de la audiencia (que mide a partir de un programa insertado en Explorer) otorga a cada sede una posición que es actualizada semanalmente. Con este criterio no se mide la calidad de los contenidos ni de la imagen de la sede en internet, pero podremos conocer la popularidad de otros portales de referencia que nos servirán para el análisis y la mejora del nuestro.

http://www.alexa.com

\section{Figura 7}

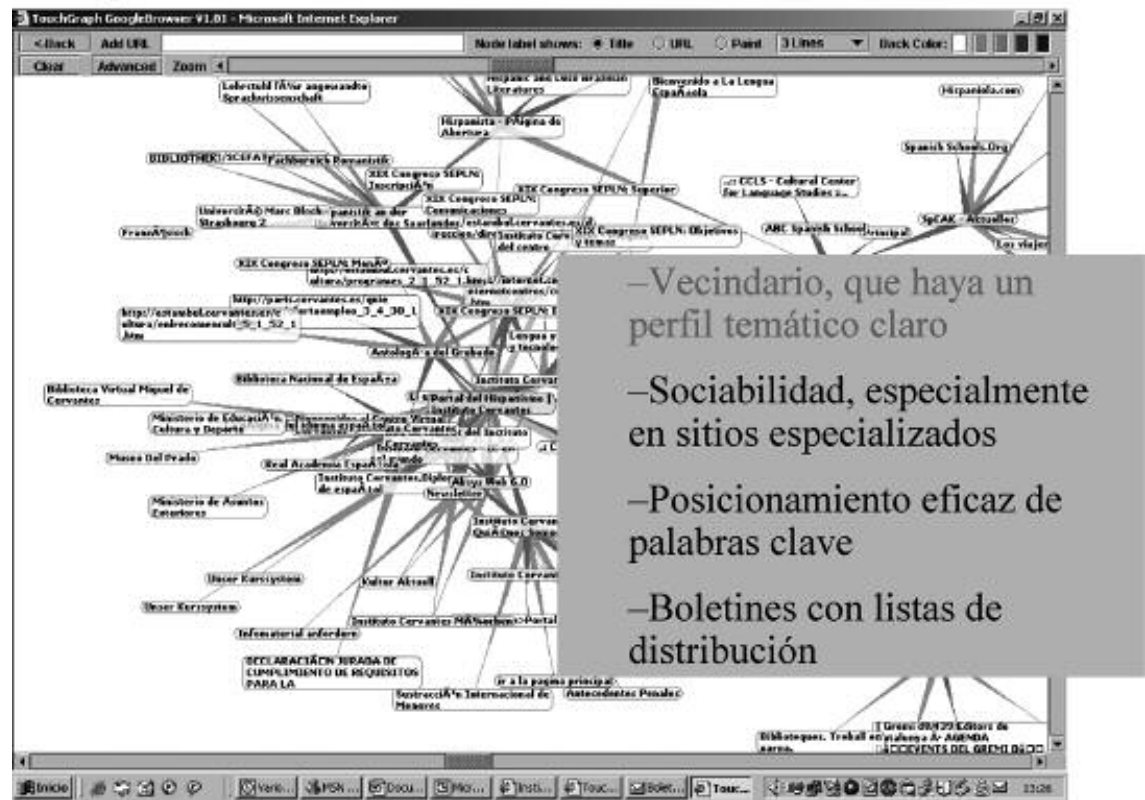

Se recomienda el uso de repertorio de herramientas especializadas o un uso avanzado de las posibilidades de los buscadores. Para medir la eficacia del posicionamiento inicial se recomienda realizar un análisis de lo que sigue:

- Control de los servicios de contenidos para conocer cuáles son los más demandados. Conviene analizar las páginas más visitadas, la frecuencia con la que son vistas y el tiempo promedio utilizado para ver cada una mediante el uso de herramientas estadísticas de medición de tráfico.

- Control de visibilidad a través de un seguimiento de las posiciones que ocupan los accesos a una página determinada en los listados que ofrecen los buscadores, a partir de consultas que realizan los usuarios mediante términos de búsqueda relacionados con los contenidos de dicha página. Es necesario haber definido un grupo de palabras claves de referencia y realizar las consultas en los buscadores. Conviene utilizar herramientas específicas y realizar un control de la eficacia de la visibilidad.

- Control del vecindario para conocer el tipo de sitios que suelen visitar los usuarios que acceden al portal analizado. Gracias a la herramienta TouchGraph de Google, se puede realizar un análisis de los enlaces de los que dispone una página determinada y que este motor de búsqueda tiene en cuenta. Esto sirve para establecer áreas de interés y para conseguir la imagen del sitio en internet (figura 7).

- Control de la sociabilidad a través de los enlaces externos realizados desde otros sitios diferentes a buscadores y directorios. Se mide el número de enlaces realizados a un sitio mediante las posibilidades que ofre-

cen algunos buscadores, como Google y Alltheweb y las herramientas estadísticas, como WebTrends, para conocer los referidores y medir la eficacia de la sociabilidad.

- Control de la integración en redes mediante la visibilidad en comunidades virtuales de usuarios, listas de distribución, boletines, etc., así como la medición estadística del impacto.

- Control de la difusión en medios a través de la propia actividad presencial del sitio y mediante el uso de las News de Google u otros medios digitales.

Resultaría útil confeccionar páginas con contenidos de calidad y diseños accesibles y usables. En general, los usuarios están mucho más interesados en los contenidos del sitio y se puede ajustar mediante un análisis exhaustivo aquellos que son más importantes para los visitantes. El estudio de las páginas por las que los usuarios acceden o abandonan el sitio es también muy útil para optimizar su arquitectura. Toda esta información sirve para proponer mejoras del posicionamiento, del diseño y de las estrategias de promoción y difusión para la obtención de la rentabilidad (figura 8).

Por último, hay que señalar que sería práctico llevar a cabo este mismo análisis de popularidad en relación a otros portales dentro del ámbito temático del sitio y que se encuentren mejor posicionados en la Red.

\section{Análisis de la eficiencia y de la rentabilidad}

Ha de contrastarse el análisis interno con el externo y verificar los datos. Los resultados que se obtienen añaden valor al sitio para futuros proyectos, mediante 
la comparación de los objetivos estratégicos.

Figura 8

Conviene realizar los siguientes contrastes:

- La actividad del sitio con las áreas de interés analizadas mediante TouchGraph para determinar el vecindario.

- Los socios y colaboradores del sitio con los referidores y portales que enlazan al mismo.

- Las palabras clave y frases de búsqueda definidas de la actividad del portal con herramientas para medir la eficacia de la visibilidad.

—Los perfiles del público objetivo definido con el origen de las visitas y las encuestas.

Se recomienda ubicar el sitio en el vecindario adecuado, añadir contenidos de calidad relacionados con sectores estratégicos del portal, la inclusión de las palabras clave y de las palabras o frases por las que los usuarios buscan el sitio en los nuevos contenidos, el intercambio de enlaces y banners con otros portales y el posicionamiento adecuado en los buscadores, todo ello llevado a cabo mediante las herramientas apropiadas para ello.

\section{Conclusión}

Todos los análisis descritos anteriormente del posicionamiento de un portal en internet sirven para facilitar las interpretaciones de los datos que permitirán posteriormente al responsable del sitio adoptar las medidas necesarias para la definición de las estrategias más eficaces y rentables.

Luis Cueto Álvarez de Sotomayor, Ministerio de Industria, Comercio y Turismo (coordinador), Director del gabinete de la subsecretaría de Industria, Comercio y Turismo. luis.cueto@min.es

Tel.: +34-913494848

Chimo Soler Herreros, Instituto Cervantes (coordinador), jefe del Departamento de Sistemas de Información.

chimo.soler@cervantes.es

Tel.: +34-914367615

Raquel Entremonzaga Ayuso, Instituto Cervantes, colaboradora externa. Marketing y estadística.

sistema13@cervantes.es

Tel.: +34-914367571

Eva Martínez, Casa de América, jefa de informática. eva@casamerica.es

Tel.: +34-915954819

Antonio Carrasco, Universidad de Alicante, subdirector



biblioteca de historia.

antonio.carrasco@cervantesvirtual.com

Tel.: +34-965909638

Isidro Aguillo, Centro de Información y Documentación Científica (Cindoc)-CSIC, responsable del Laboratorio de Internet del Cindoc.

isidro@cindoc.csic.es

Tel.: +34-915635482

Elena Fernández, Centro de Información y Documentación Científica (Cindoc)-CSIC, coordinadora de portales científicos del Cindoc.

elenaf@cindoc.csic.es

Tel.: +34-915635482

Miguel Rodríguez Artacho, Universidad Nacional de Educación a Distancia (UNED), director de Planificación de Sistemas Informáticos.

dirplansisinf@adm.uned.es

Tel.: +34-913988934

Dionisio Barrantes, Biblioteca Nacional de España, jefe del Departamento de Informática.

barrantesdbb@bne.es

Tel.: +34-915807819

Javier Espadas Bardón, Museo Thyssen-Bornemisza, jefe del Departamento de Informática.

jespadas@museothyssen.org

Tel.: +34-914203944

Rufino Ferreras Marcos, Museo Thyssen-Bornemisza, responsable del Departamento de Desarrollo Educativo. rferreras@museothyssen.org

Tel.: +34-914203944

Jaime Albert, Entidad Pública Empresarial Red.es, responsable de comunicaciones.

jaime.albert@red.es

Tel.: +34-912127625

Jesús Sanz de las Heras, Red.es/RedIris, coordinador del servicio de correo electrónico de RedIris.

jesus.heras@rediris.es

Tel.: +34-912127625 